\title{
Hamiltonian Embedding of Self-Dual Model and Equivalence with Maxwell-Chern-Simons Theory
}

\author{
R. Banerjee円 \\ Instituto de Fisica, Universidade Federal do Rio de Janeiro \\ CP 68528, 21945-970 Rio de Janeiro, RJ \\ Brazil \\ H. J. Rothe and K. D. Rothe \\ Institut für Theoretische Physik \\ Universität Heidelberg \\ Philosophenweg 16, D-69120 Heidelberg
}

\begin{abstract}
Following systematically the generalized Hamiltonian approach of Batalin and Fradkin, we demonstrate the equivalence of a self-dual model with the Maxwell-Chern-Simons theory by embedding the former second-class theory into a first-class theory.
\end{abstract}

\footnotetext{
${ }^{1}$ On sabbatical leave from S.N. Bose National Centre for Basic Sciences, DB-17, Sec 1, Salt Lake, Calcutta-700064, India
} 


\section{Introduction}

It has long been recognized that the addition of a Chern-Simons three-form to a Maxwell term in $2+1$ dimensions, will generate a topological mass for the "photon" [1]. Another model which also describes the propagation of a single massive mode in $2+1$ dimensions is the "self-dual" (SD) model originally proposed in ref. [2]. This suggested a possible equivalence between the (second-class) SD-model and the (first-class) Maxwell-Chern-Simons (MCS) theory, which has subsequently been demonstrated on a semi-classical level by Deser and Jackiw [3]. Since then there appeared a number of papers studying different aspects of this equivalence [4, 5, 6]. However, a study within the generalized canonical framework of Batalin Fradkin and Tyutin [7, 8], allowing for the systematic conversion of second-class systems into first-class ones, is lacking.

In this paper we demonstrate the above equivalence between these two models by embedding the SD-model into a gauge theory, following systematically the procedure of ref. [7]. The first class constraints and the unitarising Hamiltonian are constructed in section 2. We then show in section 3 that the partition function of the SD-model and MCS-theory are obtained for different choices of gauge, thereby establishing the claimed equivalence as a consequence of the Fradkin-Vilkovisky theorem [11]. Our conclusions are given in section 4 .

\section{BF Hamiltonian embedding}

The Lagrangian of the self-dual (SD) model [3] is given by

$$
\mathcal{L}_{S D}=\frac{1}{2} f^{\mu} f_{\mu}-\frac{1}{2 m} \epsilon_{\mu \lambda \nu} f^{\mu} \partial^{\lambda} f^{\nu}
$$

It describes a pure second-class system with three primary constraints

$$
\begin{aligned}
& T_{0}=\pi_{0} \approx 0 \\
& T_{i}=\pi_{i}+\frac{1}{2 m} \epsilon_{i j} f^{j} \approx 0
\end{aligned}
$$

and a secondary constraint

$$
T_{3}=\frac{1}{m}\left(f^{0}+\frac{1}{m} \epsilon_{i j} \partial_{i} f^{j}\right) \approx 0
$$

where $\pi_{\mu}$ are the momenta canonically conjugate to $f^{\mu}$. The secondary constraint follows from the requirement

$$
\left\{T_{0}(x), H_{T}\right\} \approx 0,
$$


where $H_{T}$ is the total Hamiltonian [13]

$$
H_{T}=H_{c}+\int d^{2} x\left(v^{0} T_{0}+\sum_{i=1}^{2} v^{i} T_{i}\right)
$$

with $v^{0}, v^{i}$ Lagrange multipliers, and $H_{c}$ the canonical Hamiltonian

$$
H_{c}=\int d^{2} x\left\{-\frac{1}{2} f^{\mu} f_{\mu}+\frac{1}{m} \epsilon_{i j} f^{0} \partial^{i} f^{j}\right\} .
$$

The non-vanishing Poisson brackets of the constraints are given by

$$
\begin{aligned}
\left\{T_{0}(x), T_{3}(y)\right\} & =-\frac{1}{m} \delta(\vec{x}-\vec{y}) \\
\left\{T_{i}(x), T_{j}(y)\right\} & =\frac{1}{m} \epsilon_{i j} \delta(\vec{x}-\vec{y}) \\
\left\{T_{i}(x), T_{3}(y)\right\} & =-\frac{1}{m^{2}} \epsilon_{i j} \partial_{j} \delta(\vec{x}-\vec{y}) .
\end{aligned}
$$

In order to establish the connection of the self-dual model with the MaxwellChern-Simons (MCS) gauge theory, we now make use of the formalism of Batalin, Fradkin and Tyutin [7, 8] to embed the second-class SD-model into a first-class theory.

We begin by converting the second-class constraints into first-class ones. We closely follow the notation of ref. [7], with the commutators in this reference being obtained from the Poisson brackets via the substitution $i\{q, p\} \rightarrow$ $[q, p]$. In the notation of [7] the first-class constraints $T_{\alpha}^{\prime}(\alpha=0,1,2,3)$ are given by

$$
T_{\alpha}^{\prime}(x)=T_{\alpha}(x)+\int d^{2} y \int d^{2} z V_{\alpha}^{\gamma}(x, y) \omega_{\gamma \beta}(y, z) \phi^{\beta}(z)
$$

where $\omega_{\gamma \beta}(x, y)$ and the structure functions $V_{\alpha}^{\gamma}(x, y)$ are restricted by the original second-class constraint algebra. The tensor $\omega_{\alpha \beta}(x, y)$ is a $c$-numerical, completely antisymmetric invertible matrix, which in our case has zero Grassman signature. The field $\phi^{\alpha}(x)$ satisfies the symplectic algebra

$$
\left\{\phi^{\alpha}(x), \phi^{\beta}(y)\right\}=\omega^{\alpha \beta}(x, y)
$$

where $\omega^{\alpha \beta}(x, y)$ is the inverse of $\omega_{\alpha \beta}(x, y)$ :

$$
\int d^{2} z \omega^{\alpha \gamma}(x, z) \omega_{\gamma \beta}(z, y)=\delta_{\beta}^{\alpha} \delta(\vec{x}-\vec{y}) .
$$

For the constraint algebra (2.7) a possible choice for $\omega_{\alpha \beta}(x, y)$ and $V_{\alpha}^{\beta}(x, y)$ is given by

$$
\begin{aligned}
& \omega_{03}(x, y)=\frac{1}{m} \delta(\vec{x}-\vec{y}) \\
& \omega_{i j}(x, y)=\frac{1}{m} \epsilon_{i j} \delta(\vec{x}-\vec{y}) \\
& \omega_{0 i}(x, y)=-\frac{1}{m^{2}} \epsilon_{i k} \partial_{k} \delta(\vec{x}-\vec{y})
\end{aligned}
$$


and

$$
\begin{aligned}
& V_{0}^{3}(x, y)=\delta(\vec{x}-\vec{y}) \\
& V_{i}^{j}(x, y)=\delta_{i}^{j} \delta(\vec{x}-\vec{y}),
\end{aligned}
$$

with $V_{\alpha}^{\beta}(x, y)=V_{\beta}^{\alpha}(y, x)$. All remaining elements of $V_{\alpha}^{\beta}$ vanish.

The inverse $\omega^{\alpha \beta}$ of $\omega_{\alpha \beta}$ is found from (2.10) and (2.11) to be

$$
\omega^{-1}(x, y)=\left(\begin{array}{cccc}
0 & 0 & 0 & -m \\
0 & 0 & -m & -\partial_{1}^{x} \\
0 & m & 0 & -\partial_{2}^{x} \\
m & -\partial_{1}^{x} & -\partial_{2}^{x} & 0
\end{array}\right) \delta(\vec{x}-\vec{y})
$$

In terms of the choice (2.11) and (2.12), the first-class constraints (2.8) read

$$
\begin{aligned}
& T_{0}^{\prime}(x)=T_{0}(x)-\frac{1}{m} \phi^{0}(x) \\
& T_{i}^{\prime}(x)=T_{i}(x)+\frac{1}{m} \epsilon_{i k} \phi^{k}(x)-\frac{1}{m^{2}} \epsilon_{i k} \partial_{k} \phi^{0}(x) \\
& T_{3}^{\prime}(x)=T_{3}(x)+\frac{1}{m} \phi^{3}(x)-\frac{1}{m^{2}} \epsilon_{k l} \partial_{l} \phi^{k}(x)
\end{aligned}
$$

One readily verifies the strongly involutive algebra

$$
\left[T_{\alpha}^{\prime}(x), T_{\beta}^{\prime}(y)\right]=0 .
$$

We next construct the corresponding involutive Hamiltonian $H^{\prime}$. Following [7], it is given by

$$
H^{\prime}=\left\{H[\varphi] \exp \int d^{2} x \frac{\overleftarrow{\partial}}{\partial \varphi^{\alpha}(x)} \phi^{\alpha}(x)\right\}_{\varphi=0}
$$

where $\varphi^{\alpha}(x)$ are real valued fields, and $H[\varphi]$ is obtained by solving the equation

$$
\frac{\partial H[\beta \varphi]}{\partial \beta}=(i)^{-2}\left[H[\beta \varphi],\left[\Omega, \int d^{2} x \bar{\Omega}_{\alpha}(x) \varphi^{\alpha}(x)\right]\right] .
$$

subject to the boundary condition $H[0]=H_{c}$. The operators $\Omega$ and $\bar{\Omega}_{\alpha}$ are defined in ref. [7], and in our particular case have the simple form

$$
\begin{aligned}
& \Omega=\int d^{2} x T_{\alpha}(x) \mathcal{C}^{\alpha}(x) \\
& \bar{\Omega}_{\alpha}(x)=\int d^{2} y \bar{V}_{\alpha}^{\gamma}(x, y) \overline{\mathcal{P}}_{\gamma}(y)
\end{aligned}
$$

where $\bar{V}_{\alpha}^{\gamma}$ is the inverse of $V_{\alpha}^{\gamma}$, as given by

$$
\begin{aligned}
& \bar{V}_{0}^{3}(x, y)=\bar{V}_{3}^{0}(\vec{y}, \vec{x})=\delta(\vec{x}-\vec{y}) \\
& \bar{V}_{1}^{1}(x, y)=\bar{V}_{2}^{2}(x, y)=\delta(\vec{x}-\vec{y})
\end{aligned}
$$


with the remaining matrix elements vanishing. The fields $\mathcal{C}^{\alpha}(x)$ and $\overline{\mathcal{P}}_{\beta}(x)$ represents a canonical ghost pair with opposite Grassman parity to that of the constraints $T_{\alpha}$ :

$$
\left[\mathcal{C}^{\alpha}(x), \overline{\mathcal{P}}_{\beta}(y)\right]=i \delta_{\beta}^{\alpha} \delta(\vec{x}-\vec{y}) .
$$

Defining the generating functional

$$
L[\varphi]=\int d^{2} x d^{2} y \varphi^{\alpha}(x) V_{\alpha}^{\gamma}(x, y) T_{\gamma}(y)
$$

the solution to (2.17) for $\beta=1$ can be written in the form

$$
H[\varphi]=e^{i L[\varphi]} H_{c} e^{-i L[\varphi]} .
$$

Expanding the exponentials in powers of $L[\varphi]$, one finds that the series truncates after the third term, with the result

$$
\begin{aligned}
H[\varphi]= & H_{c}+\left[i L, H_{c}\right]+\frac{1}{2 !}\left[i L,\left[i L, H_{c}\right]\right] \\
= & H_{c}-\int d^{2} z\left\{m \varphi^{3}(z) T_{3}(z)+\frac{1}{m} \varphi^{k}(z)\left(\epsilon_{k l} \partial_{l} f^{0}(z)-f^{k}(z)\right)\right\} \\
& -\frac{1}{2 !} \int d^{2} z\left\{\varphi^{3}(z) \varphi^{3}(z)-\varphi^{k}(z) \varphi^{k}(z)-\frac{2}{m} \epsilon_{k l} \varphi^{3}(z) \partial_{l} \varphi^{k}(z)\right\}
\end{aligned}
$$

where $k$ and $l$ take the values 1,2. From here, and the definition (2.16) one obtains for the involutive Hamiltonian

$$
\begin{aligned}
H^{\prime}= & H_{c}-\int d^{2} z m \phi^{3}(z) T_{3}^{\prime}(z)+\frac{1}{2} \int d^{2} z\left(\phi^{k}(z) \phi^{k}(z)+\phi^{3}(z) \phi^{3}(z)\right) \\
& -\int d^{2} z \phi^{k}(z)\left(\frac{1}{m} \epsilon_{k l} \partial_{l} f^{0}(z)-f^{k}(z)\right)
\end{aligned}
$$

with the strongly involutive property

$$
\left[H^{\prime}, T_{\alpha}^{\prime}(x)\right]=0 \text {. }
$$

One readily checks that the involutive Hamiltonian (2.24) is obtained from the canonical one by a simple translation in the fields

$$
H^{\prime}=H_{c}\left[f^{i}+\phi^{i}, f^{0}+\phi^{3}\right]
$$

This property will be useful in the forthcoming analysis.

We next proceed to construct the BRST Hamiltonian and the corresponding partition function. From the work of ref. [0] it is readily seen that

$$
H^{\prime}=H_{\mathrm{BRST}},
$$


since $H^{\prime}$ is strongly involutive. The remaining step concerns the construction of the unitarizing Hamiltonian $H_{U}$ as defined in [7]

$$
H_{U}=H_{\mathrm{BRST}}+\frac{1}{i}[\Psi, Q]
$$

where the nilpotent BRST charge $Q$ and the fermion function $\Psi$ are given by

$$
\begin{aligned}
& Q=\int d^{2} z\left(T_{\alpha}^{\prime} C^{\alpha}+p_{\alpha} \mathcal{P}^{\alpha}\right) \\
& \Psi=\int d^{2} z\left(\bar{C}_{\alpha} \chi^{\alpha}+\overline{\mathcal{P}}_{\alpha} q^{\alpha}\right)
\end{aligned}
$$

and where $\left(q^{\alpha}, p_{\alpha}\right)$ are canonically conjugate multiplier fields, and $\chi^{\alpha}$ are gauge fixing functions having the same statistics as the constraints, and are required to satisfy

$$
\operatorname{det}\left\{\chi^{\alpha}, T_{\beta}^{\prime}\right\} \neq 0
$$

This completes our analysis on the operator level.

We now consider the partition function corresponding to the unitarizing Hamiltonian (2.28):

$$
Z=\int[D \mu](\operatorname{det} \omega)^{-1 / 2} e^{i S_{U}}
$$

where

$$
S_{U}=\int d^{3} x\left\{\pi^{\mu} \dot{f}^{\mu}+\frac{1}{2} \phi^{\alpha} \omega_{\alpha \beta} \dot{\phi}^{\beta}+p_{\alpha} \dot{q}^{\alpha}+\mathcal{P}_{\alpha} \dot{\bar{C}}^{\alpha}+C_{\alpha} \dot{\overline{\mathcal{P}}}^{\alpha}-H^{\prime}-\frac{1}{i}[\Psi, Q]\right\}
$$

and the integration measure $[d \mu]$ involves all the fields appearing in the exponent. The second term in (2.33) is due to the symplectic algebra (2.9) satisfied by the $\phi$-fields, and the field independence of the corresponding symplectic metric $\omega^{\alpha \beta}$ [9]. This term (including det $\omega$ ) can in principle be put into a standard canonical form via a Darboux transformation [10]. In the present case it is, however, more convenient. to proceed directly from (2.33).

In the following we consider gauge functions $\chi^{\alpha}$ which do not depend on the multipliers $q^{\alpha}, p_{\alpha}$. By suitably rescaling the fields [11, 12 $\chi^{\alpha} \rightarrow$ $\chi^{\alpha} / \beta, p_{\alpha} \rightarrow \beta p_{\alpha}, \bar{C}_{\alpha} \rightarrow \beta \bar{C}_{\alpha}$ (the superjacobian of this transformation is unity) and taking $\beta \rightarrow 0$, one can carry out the integrals over the ghost variables and multiplier fields, with the result

$$
Z=\int D \pi_{\mu} D f^{\mu} D \phi^{\alpha} \delta[\chi] \delta\left[T^{\prime}\right] \operatorname{det}\left[\chi^{\alpha}, T_{\beta}^{\prime}\right] e^{i \int d^{3} x\left\{\pi_{\mu} \dot{f}^{\mu}+\frac{1}{2} \phi^{\alpha} \omega_{\alpha \beta} \dot{\phi}^{\beta}-\mathcal{H}_{c}\left[f^{i}+\phi^{i}, f^{0}+\phi^{3}\right]\right\}}
$$

where we have used (2.26) and have dropped $(\operatorname{det} \omega)^{-1 / 2}$, which is just an (irrelevant) constant. 


\section{Equivalence of SD and MCS model}

In this section we prove the equivalence of the self-dual [3] and the MaxwellChern-Simons theory, by using the Fradkin-Vilkovisky theorem [11] stating the gauge independence of the partition function (2.34). To start out with, we choose a class of gauges resticted by the two gauge conditions

$$
\chi^{i}=\phi^{i}-\frac{1}{m} \partial_{i} \phi^{0} \approx 0, \quad i=1,2
$$

The other two conditions $\chi^{0} \approx \chi^{3} \approx 0$ are still arbitrary. Using (3.1), all momentum integrals as well as $\phi^{1}, \phi^{2}$ and $\phi^{3}$ integrals can be done trivially, leading to the result

$$
\begin{aligned}
& Z=\int D f^{\mu} D \phi^{0} \delta\left[\chi^{0}\right] \delta\left[\chi^{3}\right] \\
& \times \exp i \int\left\{-\frac{1}{m^{2}} \phi^{0} \epsilon_{i j} \partial_{i} \dot{f}^{j}+\frac{1}{m} f^{i} \partial_{i} \phi^{0}\right. \\
& \left.+\frac{1}{m} f^{1} \dot{f}^{2}-\frac{1}{2 m^{2}}\left(\epsilon_{i j} \partial_{i} f^{j}\right)^{2}-\frac{1}{2} \vec{f}^{2}-\frac{1}{2 m^{2}}\left(\partial_{i} \phi^{0}\right)^{2}\right\}
\end{aligned}
$$

where the property $\epsilon_{i j} \partial_{i} \phi^{j}=0$, following from (3.1) has been used. Note that at least one of the gauge conditions $\chi^{0} \approx \chi^{3} \approx 0$ must involve $f^{0}$ in order to render the $f^{0}$-integral finite.

a) Recovery of the SD-model in the unitary gauge

Choosing for the remaining gauge conditions

$$
\begin{gathered}
\chi^{0}=\phi^{0} \approx 0 \\
\chi^{3}=T^{3}=f^{0}+\frac{1}{m} \epsilon_{i j} \partial_{i} f^{j} \approx 0
\end{gathered}
$$

one is led to the partition function of the self-dual model,

$$
Z=\int D f^{\mu} \delta\left[f^{0}+\frac{1}{m} \epsilon_{i j} \partial_{i} f^{j}\right] e^{i \int \mathcal{L}_{S D}}
$$

Note that to arrive at this expression, repeated use has been made of the gauge condition (3.4). This gauge condition is just the original second-class constraint $T^{3} \approx 0$. Similarly from $\delta\left[T^{\prime 0}\right]$ in $(2.34)$ we see that the condition (3.3) can also be viewed as the original second-class constraint $T^{0}=\pi^{0} \approx 0$. For this reason the above gauge is referred to in the literature [7, 8] as the "unitary" gauge. 
b) Recovery of the MCS model in the Coulomb-like gauge

Consider the gauge condition [6]

$$
\chi^{0}, \chi^{3}: f^{i}-\frac{1}{m} \epsilon_{i j} \partial_{j} f^{0} \approx 0
$$

Since this gauge implies $\partial_{i} f^{i}=0$, we refer to it as the Coulomb-like gauge. Using (3.4), the partition function (3.2) can be put into the form

$$
\begin{aligned}
& Z=\int D f^{\mu} D \phi^{0} \delta\left[f^{i}-\frac{1}{m} \epsilon_{i j} \partial_{j} f^{0}\right] \times \\
& \times \exp i \int d^{3} x\left\{-\frac{1}{m^{2}} \phi^{0} \epsilon_{i j} \partial_{i} \dot{f}^{j}+\frac{1}{2 m^{2}} \phi^{0} \vec{\nabla}^{2} \phi^{0}\right. \\
& \left.+\frac{1}{m} f^{1} \dot{f}^{2}-\frac{1}{2 m^{2}}\left(\epsilon_{i j} \partial_{i} f^{j}\right)^{2}-\frac{1}{2 m^{2}}\left(\epsilon_{i j} \partial_{j} f^{0}\right)^{2}\right\}
\end{aligned}
$$

Performing the Gaussian $\phi^{0}$-integration we find

$$
\begin{aligned}
Z= & \int D f^{\mu} \delta\left[f^{i}-\frac{1}{m} \epsilon_{i j} \partial_{j} f^{0}\right] \times \\
& \exp i \int d^{3} x\left\{-\frac{1}{2 m^{2}}\left(\epsilon_{i j} \partial_{i} \dot{f}^{j}\right) \frac{1}{\vec{\nabla}^{2}}\left(\epsilon_{k l} \partial_{k} \dot{f}^{l}\right)\right. \\
& \left.+\frac{1}{2 m} \epsilon_{i j} f^{i} \dot{f}^{j}-\frac{1}{2 m^{2}}\left(\epsilon_{i j} \partial_{i} f^{j}\right)^{2}-\frac{1}{2 m^{2}}\left(\epsilon_{i j} \partial_{j} f^{0}\right)^{2}\right\}
\end{aligned}
$$

Making repeated use of the gauge condition, the partition function can be put into the manifestly covariant form

$$
Z=\int D f^{\mu} \delta\left[f^{i}-\frac{1}{m} \epsilon_{i j} \partial_{j} f^{0}\right] e^{i \int d^{3} x \mathcal{L}_{\mathrm{MCS}}}
$$

where $\mathcal{L}_{\mathrm{MCS}}$ is the Maxwell-Chern-Simons Lagrangian

$$
\mathcal{L}_{\mathrm{MCS}}=-\frac{1}{4 m^{2}} f_{\mu \nu} f^{\mu \nu}-\frac{1}{2 m} \epsilon_{\mu \nu \lambda} f^{\mu} \partial^{\nu} f^{\lambda}
$$

It is simple to see that the gauge condition appearing in (3.9) is equivalent to the Coulomb gauge and Gauss law in that gauge. This completes the proof of the equivalence of the self-dual model and the Maxwell-Chern-Simons theory within the BF framework.

\section{Conclusion}

The equivalence of the self-dual (SD) model of ref. [2] and the MaxwellChern-Simons theory has been discussed using different approaches [3, 4, 5, 6]. It was the objective of this paper to demonstrate this equivalence by suitably extending the phase space of the SD-model, following systematically the 
procedure of ref. [7]. This involved the conversion of the four second-class constraints of the SD model into the first-class ones, and the corresponding construction of the unitarising Hamiltonian. The gauge-invariant partition function associated with this Hamiltonian was shown to reduce to that of the original second-class SD-model in the "unitary gauge", and to the MCS theory in a Coulomb-like gauge. It is interesting to note that the field $f^{\mu}$ in the embedded partition function (2.34) played the role of either the fundamental field of the SD-model or the gauge potential of the MCS theory, in the unitary and Coulomb-like gauge, respectively.

Finally, we wish to point out that the equivalence investigated here has proven useful in the study of abelian bosonization in $2+1$ dimensions of massive fermionic models [14], 15].

\section{Acknowledgement}

One of the authors (R.B.) would like to thank the CNPq for financial support.

\section{References}

[1] S. Deser, R. Jackiw, and S. Templeton, Ann. Phys. 140 (1982) 372.

[2] P. K. Townsend, K. Pilch, and P. Van Nieuwenhuizen, Phys. Lett. 136B (1984) 38.

[3] S. Deser and R. Jackiw, Phys. Lett. 139B (1984) 371.

[4] R. Gianvittorio, A. Restuccia, and J. Stephany, Mod. Phys. Lett. A6 (1991) 2121.

[5] R. Banerjee, H. J. Rothe, and K. D. Rothe, Phys. Rev. D52 (1995) 3750 .

[6] R. Banerjee and H. J. Rothe, Nucl. Phys. B447 (1995) 183.

[7] I. A. Batalin and E. S. Fradkin, Nucl. Phys. B279 (1987) 514.

[8] I. A. Batalin and I. V. Tyutin, Int. J. Mod. Phys. A6 (1991) 3255.

[9] R. Amorim, Z.Phys. C67 (1995) 695.

[10] R. Jackiw, "Constrained Quantization without Tears" ("Diverse Topics in Theoretical and Mathematical Physics", World-Scientific, Singapore, 1995.)

[11] E. S. Fradkin and G. Vilkovisky, Phys. Lett. B55 91975) 224. 
[12] M. Henneaux, Phys. Rep. 126 (1985) 1.

[13] P. A. M. Dirac, "Lectures on Quantum Mechanics", Belfer Graduate School of Science, Yeshiva University, N.Y. 1964.

[14] E. Fradkin and F. Schaposnik, Phys. Lett. B338 (1994) 253.

[15] R. Banerjee, Phys. Lett. B358 (1995) 297; Nucl. Phys. B465 (1996) 157. 